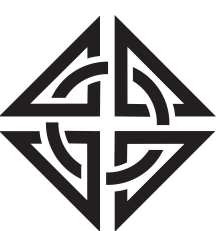

SCIENTIA
Sharif University of Technology

Scientia Iranica

Transactions D: Computer Science 83 Engineering and Electrical Engineering www.scientiairanica.com

\title{
Guaranteed cost control of uncertain discrete time systems subjected to actuator saturation via homogenous polynomial Lyapunov function
}

\author{
S.M. Nashit Arshad ${ }^{a}$, K. Mazhar ${ }^{a}$, M. Umer Khan ${ }^{a}$ and S.M. Usman Hashmi ${ }^{b, *}$ \\ a. Department of Mechatronics, Air University, Islamabad, Pakistan. \\ b. Department of Electronics Engineering, Iqra University, Islamabad, Pakistan.
}

Received 17 December 2014; received in revised form 30 June 2015; accepted 4 January 2016

\section{KEYWORDS \\ Convex optimization; \\ Lyapunov function; \\ Uncertainty; \\ Discreet time control.}

\begin{abstract}
Estimation of an optimal controller is a fundamental problem in control engineering and is widely known as Optimization. Numerous computation and numerical techniques have been evolved during the past years for the estimation of the optimal solution. Optimal control of a discrete-time system is concerned with optimizing a given objective function using "Homogenous Polynomial Lyapunov Function (HPLF)". This research focuses upon the design of optimal Guaranteed Cost Controller (GCC) for discretetime uncertain system using HPLF. The uncertainties are assumed to be norm-bounded uncertainties. The effect of actuator saturation is also incorporated in the system. Sufficient conditions for the existence of HPLF are derived in terms of Linear Matrix Inequalities (LMI). The LMI approach has the advantage that it can be solved efficiently using Convex Optimization. LMI's combined with HPLF helps to design the guaranteed cost controller which minimizes the cost by minimizing cost function. Furthermore, the state trajectories and the invariant set are also shown for the observation of the overall performance.
\end{abstract}

(C) 2016 Sharif University of Technology. All rights reserved.

\section{Introduction}

Virtually, all control actuation devices are subjected to magnitude or rate limits, i.e. current, torque, flow, force, voltage, etc. This nonlinearity causes the control system to operate under constraints on the magnitude of the control input. For this reason, the formulation of many fundamental control problems, including optimal control and controllability, reflects the constraint imposed by actuator saturation $[1,2]$. Performance degradation takes place when actuator saturates. In extreme cases, stability can be jeopardizing.

*. Corresponding author. Tel.:+92-333-5376480 E-mail addresses: nashit@iqraisb.edu.pk (S.M. Nashit Arshad); kamal.mazhar88@gmail.com (K. Mazhar); umer.khan@mail.au.edu.pk (M. Umer Khan); usman16288@yahoo.com (S.M. Usman Hashmi)
In control system literature, robust controller for linear systems with parameter uncertainty has drawn much considerable attention [3,4]. A lot of research has been carried out to promise not only robustness but also stability of the system. In this research, asymptotic stability is discussed for the solutions of a discrete time system $[2,5]$.

In the theory of optimal control, Lyapunov functions [6-8] are the scalar functions that are used widely to prove the stability of a dynamical system. For instance, quadratic Lyapunov function, which has drawn valuable attention, serves for the systems with state variables involving the existence of linear matrix inequalities $[9,10]$. More general classes of Lyapunov functions have been considered, i.e. polyhedral Lyapunov function [11], piecewise quadratic Lyapunov function [12], and saturation dependent Lyapunov functions [13]. However, HPLF as a viable alternative 
to the above classes of Lyapunov functions in a way that it uses a nonlinear Lyapunov function to optimize the control sequence by keeping the system less conservative and providing the enlargement/enhancement to the invariant set $[14,15]$.

Optimization and control problems are highly intertwined. If a control sequence, also known as control law, has to be chosen, control parameters and control input signal are interpreted as decision variables of an optimization problem. In recent years, linear matrix inequalities have been considered to be a powerful tool for approaching the control problem that appears difficult to solve in analytical fashion $[3,16]$.

When controlling a plant, it requires not only making the system asymptotically stable but also promising an adequate level of performance. One approach to this problem is called the Guaranteed Cost Controller (GCC) [5]. Guaranteed cost controller formulates optimal control law using quadratic Lyapunov function which gives an upper bound to the closed loop values of the performance index and thus the system degradation incurred by the model parameter uncertainties is guaranteed to be less than a bound. The implementation of guaranteed cost control using quadratic Lyapunov function has drawn much attention of research community and many approaches have been proposed and developed $[12,17,18]$. $\mathrm{Yu}$ et al. [19] proposed LMI approach for the GCC design and the design problem of optimal guaranteed cost controller which minimizes the corresponding guaranteed cost. This approach [1] was formulated as convex optimization problem with LMI constraints.

This research focuses on guaranteed cost controller problem for uncertain discrete-time system subject to actuator saturation via HPLF. It is widely known that quadratic functions lead to conservative estimates of the robust stability margin [20]. Hence, non-quadratic homogenous Lyapunov functions are addressed. The problem of constructing homogenous Lyapunov function is dealt by means of recently developed convex optimization technique using linear matrix inequalities. Actuator saturation and uncertainties are also taken into account while considering the linear constraints. The system matrices and the state vectors are transformed using power transformation introduced in $[3,21,22]$. The proposed research transforms the saturation non-linearity into a convex polytope of linear system and then formulates a convex optimization problem with the constraint given by a set of LMIs.

This research paper is divided into 5 Sections, beginning with an Introduction. Detailed views of the preliminaries are presented in Section 2 and the problem statement is presented in Section 3. The novelty of the research and the proposed idea are presented in Section 4. Section 5 deals with the examples, results, and conclusion.

\section{Preliminaries}

This section provides the preliminaries required for the development of the proposed scheme.

\subsection{Power transformation of state vector}

Definition 1: [3] Consider a vector $x \in \mathbb{R}^{n}$. Power transformation of the degree $p$ is a nonlinear change of coordinates that creates a vector $x^{\{p\}}$ of all the monomials of degree $p$ and can be produced from the original $x$ vector:

$$
x_{l}^{\{p\}}:=x_{1}^{p_{l 1}} x_{2}^{p_{l 2}} \ldots . . x_{n}^{p_{l n}} \quad l=1, \ldots ., m,
$$

with:

$$
m=\left(\begin{array}{c}
n+p-1 \\
p
\end{array}\right)
$$

ordered by the degree of the leftmost variable.

Note that with this definition $x^{\{0\}}=1$, and $x^{\{1\}}=x$.

The followings are examples to illustrate the above concept:

1. The most basic form is $n=p=2 \Rightarrow m=3$

$$
x=\left[\begin{array}{l}
x_{1} \\
x_{2}
\end{array}\right] \Rightarrow x^{\{2\}}=\left[\begin{array}{c}
x_{1}^{2} \\
x_{1} x_{2} \\
x_{2}^{2}
\end{array}\right] .
$$

2. $n=3, p=3 \Rightarrow m=10$

$$
x=\left[\begin{array}{c}
x_{1} \\
x_{2} \\
x_{3}
\end{array}\right] \Rightarrow x^{\{3\}}=\left[\begin{array}{c}
x_{1}^{3} \\
x_{1}^{2} x_{2} \\
x_{1}^{3} x_{3} \\
x_{1} x_{2}^{2} \\
x_{1} x_{2} x_{3} \\
x_{1} x_{3}^{2} \\
x_{2}^{3} \\
x_{2}^{3} x_{3} \\
x_{2} x_{3}^{2} \\
x_{3}^{3}
\end{array}\right] .
$$

\subsection{Power transformation of system matrices}

For a generic system $\dot{x}=A(t) x(t)$, let us introduce the extended matrix $A_{[m]}(t) \in \mathbb{R}^{n^{m} \times n^{m}}$, defined as:

$$
\frac{d}{d t} x^{\{m\}}=\frac{\partial x^{\{m\}}}{\partial x} A x=A_{[m]} x^{\{m\}} .
$$

An expression of the extended matrix is provided in terms of knocker product as: 
Theorem 1: $\quad[1,23]$ Let $x^{\{m\}}=x \otimes x \otimes \cdots \otimes x$, where the number of Kronecker products is denoted by $m$, and $K_{m} \in \mathbb{R}^{n^{m}} \times d$ be the matrix satisfying:

$$
x^{[m]}=K_{m} x^{\{m\}} .
$$

Then, the extended matrix $A_{[m]}$ of $A \in \mathbb{R}^{n^{m} \times n^{m}}$ is given by:

$$
A_{[m]}=\left(K_{m}^{T} K_{m}\right)^{-1} K_{m}^{T}\left(\sum_{i=0}^{m-1} I_{n^{m-1-i}} \otimes A \otimes I_{n^{i}}\right) K_{m}
$$

Moreover, the dimension of $d$ is given by:

$$
d=\frac{(n+m-1) !}{(n-1) ! m !} \text {. }
$$

Proof: Let $A_{[m]} \in \mathbb{R}^{n^{m} \times n^{m}}$ be the matrix satisfying:

$$
\frac{\partial x^{\{m\}}}{\partial t} A x=A_{[m]} x^{\{m\}} .
$$

From the properties of Kronecker product, it turns out that:

$$
\frac{\partial x^{\{m\}}}{\partial t}=\sum_{i=0}^{m-1} x^{\{m-1-i\}} \otimes I_{n} \otimes x^{\{i\}},
$$

and:

$$
\begin{aligned}
\left(x^{\{m-1-i\}} \otimes I_{n} \otimes x^{\{i\}}\right) & A x=x^{\{m-1-i\}} \otimes A x \otimes x^{\{i\}} \\
& =\left(I_{n^{m-1-i} \otimes A \otimes I_{n^{i}}}\right) x^{\{m\}} .
\end{aligned}
$$

Therefore, matrix $A_{[m]}$ admits the following expression:

$$
A_{[m]}=\sum_{i=0}^{m-1} I_{n^{m-1-i}} \otimes A \otimes I_{n^{i}} .
$$

Now, from Eqs. (3) and (6), we have:

$$
K_{m} \frac{\partial x^{\{m\}}}{\partial t} A x=A_{[m]} K_{m} x^{\{m\}} .
$$

Given $A \in \mathbb{R}^{n \times n}$ with $x(k+1)=A x(k)$, there exists a matrix:

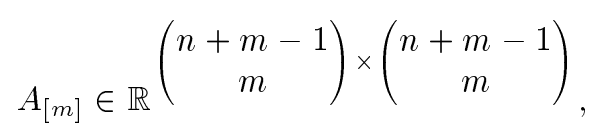

and the operator that maps $A$ into $A_{[m]}$ is linear. To clarify the meaning of $A_{[m]}$, consider the following example where $n=m=2$ :

$$
A=\left[\begin{array}{ll}
a_{11} & a_{12} \\
a_{21} & a_{22}
\end{array}\right] \Rightarrow A_{[2]}=\left[\begin{array}{ccc}
2 a_{11} & 2 a_{12} & 0 \\
a_{21} & a_{11}+a_{22} & a_{12} \\
0 & 2 a_{21} & 2 a_{22}
\end{array}\right]
$$

\section{Problem statement}

\subsection{Guaranteed cost controller}

Consider a discrete time system:

$$
x(k+1)=A x(k)+B u(k),
$$

with the initial condition:

$$
x(0)=x_{0} .
$$

The system states vector is represented by $x(k) \in \mathbb{R}^{n}$ and the control input vector is given by $u(k) \in \mathbb{R}^{m}$.

Characterization of the set of all stabilizing controllers is central in practice because stability is the minimal requirement for a closed loop system. Generally, to satisfy some performance requirements, it is necessary to define a performance criterion and to pick, among all the stabilizing controllers, a controller which leads to a satisfactory closed loop system behavior. Thus, the required performance can be achieved by associating System (8) with the following cost function [1].

$$
J=\sum_{k=0}^{\infty}\left[x^{T}(k) Q x(k)+u^{T}(k) R u(k)\right],
$$

where $Q \in \mathbb{R}^{n \times n}$ and $R \in \mathbb{R}^{n \times n}$ are given positive semi-definite symmetric matrices and $J$ is called the Guaranteed Cost.

Lemma 1: [19] A state feedback control law $u=K x$ is said to be quadratically guaranteed cost controller of System (8) with Cost Function (9) if there exists a symmetric positive definite matrix $P \in \mathbb{R}^{n \times n}$ such that:

$$
[A+B K]^{T} P[A+B K]-P+Q+K^{T} R K<0,
$$

where $P$ is called the guaranteed cost matrix.

Now, consider the discrete time system with norm bounded uncertainty and saturation:

$$
x(k+1)=(A+\Delta A) x(k)+(B+\Delta B) u(k) .
$$

The system states vector is represented by $x(k) \in \mathbb{R}^{n}$ and the control input vector is given by $u(k) \in \mathbb{R}^{m}$. $A$ and $B$ are known as constant real matrices of the appropriate dimension. The considered parametric uncertainties are of the form:

$$
\left[\begin{array}{cc}
\Delta A & \Delta B
\end{array}\right]=D\left[\begin{array}{ll}
E_{1} & E_{2}
\end{array}\right]
$$

where $D, E_{1}$ and $E_{2}$ are matrices of the uncertainty with compatible dimension.

The control input $u$ in System (8) is subjected to the following constraint:

$$
-\tilde{u}_{i} \leq u_{i} \leq \tilde{u}_{i}
$$


where $u_{i}$ is the $i$ th element in the control input $u . \tilde{u}_{i}$, $i=1,2, \cdots, m$, is the saturating magnitude. Thus, $u(k)$ can be described by $\operatorname{diag}\left\{\tilde{u}_{i}\right\} \sigma(u)$ without loss of generality [19]. The function $\sigma: \mathbb{R}^{m} \rightarrow \mathbb{R}^{m}$ is the standard saturation function defined as follows:

$$
\sigma(u)=\left[\sigma\left(u_{1}\right) \sigma\left(u_{2}\right) \cdots \sigma\left(u_{m}\right)\right]^{T} .
$$

For saturation levels $i=1,2, \cdots, m$ :

$$
\sigma\left(u_{i}\right)=\operatorname{sign}\left(u_{i}\right) \min \left\{1,\left|u_{i}\right|\right\} .
$$

Let $\nu$ be the set of diagonal matrices of $m \times m$ whose diagonal elements are either 1 or 0 . There exist $2^{m}$ elements in $\nu$. Suppose that each element of $\nu$ is labeled as $D_{i}, i=1,2, \cdots, 2^{m}$. Then, $D=\left\{D_{i}: i \in\left[1,2^{m}\right]\right\}$. Denotes $\bar{D}_{i}=I-D_{i}$. Clearly, $\bar{D}_{i}$ is also an element of $\nu$ if $D_{i} \in \nu$.

Lemma 2: [2] Let $K, H \in \mathbb{R}^{m \times n}$ be the known matrices. For $x(k) \in \mathbb{R}^{n}$, if $\|H x\|_{\infty} \leq 1$, then:

$$
\sigma(K x) \in \operatorname{co}\left\{D_{i} K x+\bar{D}_{i} H x: i \in\left[1,2, \cdots, 2^{m}\right]\right\},
$$

where $\operatorname{co}\{$.$\} represents the set of a convex hulls. System$ (11) now becomes:

$$
\begin{aligned}
& x(k+1)=[(A+\Delta A) \\
& \left.\quad+(B+\Delta B) \operatorname{diag}\left\{\tilde{u}_{i}\right\}\left(D_{i} K+\bar{D}_{i} H\right)\right] x(k) .
\end{aligned}
$$

For simplicity, we have assumed $(A+\Delta A)=A^{\prime}$ and $(B+\Delta B)=B^{\prime}$.

Lemma 3: A state feedback law $u=K x$ is said to be quadratically guaranteed cost controller of System (14) with Cost Function (9), if there exists a symmetric positive define matrix $P \in \mathbb{R}^{n \times n}$ such that:

$$
\begin{aligned}
& {\left[A^{\prime}+B^{\prime} \operatorname{diag}\left\{\tilde{u}_{i}\right\}\left(D_{i} K+\bar{D}_{i} H\right)\right]^{T}} \\
& \left.\quad P\left[A^{\prime}+B^{\prime}\right) \operatorname{diag}\left\{\tilde{u}_{i}\right\}\left(D_{i} K+\bar{D}_{i} H\right)\right] \\
& \quad-P+Q+K^{T} R K<0 .
\end{aligned}
$$

Lemma 4: If $u=K x$ is quadratically guaranteed cost controller of System (14) with Cost Function (9), then the closed loop system:

$$
x(k+1)=\left[A^{\prime}+B^{\prime} \operatorname{diag}\left\{\tilde{u}_{i}\right\}\left(D_{i} K+\bar{D}_{i} H\right)\right] x(k),
$$

is quadratically stable, and the cost function value of the closed loop system is not more than:

$$
J^{*}=x_{0}^{\{p\}^{T}} P x_{0}^{\{p\}} .
$$

The objective of this research is to develop a procedure for the development of optimal guaranteed cost controller for System (14) subjected to Input Constraint (13) via HPLF.

\section{Main result}

In this section, development of the homogenous polynomial Lyapunov function is presented.

\subsection{Homogeneous polynomial Lyapunov function}

$$
V(x(k))=x^{T}(k) P x(k),
$$

where $P$ is positive definite real symmetric matrix.

The Lyapunov stability of the discrete time System (14) is given by:

$$
\nabla V(x(k))=x^{T}(k)\left(A^{T} P A-P\right) x(k),
$$

since $\nabla V(x(k))$ is chosen to be positive definite. Therefore:

$$
Q=-\left(A^{T} P A-P\right)=\text { positive definite. }
$$

Hence, for the asymptotic stability of the discrete time System (8), it is sufficient that $Q$ be positive definite.

Now, consider the case where Lyapunov function is a form of homogenous polynomial. The construction of Lyapunov function providing stability of System (14) is restricted to a special class of Lyapunov functions: the homogenous polynomial form of degree $2 m$ denoted by $v_{2 m}(x)$. More specially, the aim is to find $v_{2 m}(x)$ such that:

(i) $v_{2 m}(x)>0$ for all $x \neq 0$,

(ii) $\dot{v}_{2 m}(x)<0$ for all $x \neq 0$,

where $v_{2 m}(x)$ has the form:

$$
v_{2 m}(x)=x^{\{p\}^{T}} P x^{\{p\}},
$$

where $2 m$ represents the degree of polynomial and $x^{\{p\}} \in \mathbb{R}^{d}$ represents the base vector of homogenous form of degree $m$. The number of state variables are given by $n$ and the dimension $d$ of $x^{\{p\}}$ can be checked by Eq. (5).

For the case $m=2$, the HPLF is given by:

$$
\begin{aligned}
& v_{2 m}(x)=x^{\{2\}^{T}} P x^{\{2\}}, \\
& v_{2 m}(x)=\left[\begin{array}{c}
x_{1}^{2} \\
x_{1} x_{2} \\
x_{2}^{2}
\end{array}\right]^{T}\left[\begin{array}{lll}
p_{11} & p_{12} & p_{13} \\
p_{21} & p_{22} & p_{23} \\
p_{31} & p_{32} & p_{33}
\end{array}\right]\left[\begin{array}{c}
x_{1}^{2} \\
x_{1} x_{2} \\
x_{2}^{2}
\end{array}\right] .
\end{aligned}
$$

For the computation of the HPLF, there exists a transformation matrix that satisfies Theorem 1. For a system having two states variables, the transformation matrix is given by Eq. (7). After the computation of the extended base vector $x^{\{2\}}$ and the transformation matrix $A_{[p]}$, an optimal guaranteed cost controller can be developed. 


\subsection{Problem formulation using linear matrix inequalities}

The constraints which were developed in the earlier section can now be posed in the form of linear matrix inequalities. These LMIs are then posed as a convex optimization problem and solved using $c v x$ Matlab ${ }^{\circledR}$ Toolbox.

Theorem 2: For a symmetric positive definite matrix $P \in \mathbb{R}^{n \times n}$ and a scalar $\rho \in \mathbb{R}^{+}$, consider the ellipsoid $\varepsilon(P, \rho):=\left\{x \in \mathbb{R}^{n}: x^{\{m\}^{T}} P x^{\{m\}} \leq \rho\right\}$. If there exists a positive definite extended matrix $G_{[m]} \in$ $\mathbb{R}^{n \times n}$ and $V_{[m]}, W_{[m]} \in \mathbb{R}^{m \times n}$. For simplicity, $G, V$ and $W$ are used instead of $G_{[m]}, V_{[m]}$ and $W_{[m]}[23,24]$.

$$
\begin{aligned}
& {\left[\begin{array}{cccc}
-G & G & W^{T} & X^{T} \\
G & -\rho Q^{-1} & 0 & 0 \\
W & 0 & -\rho R^{-1} & 0 \\
X & 0 & 0 & -G
\end{array}\right]<0} \\
& {\left[\begin{array}{cc}
1 & v_{i} \\
v_{i}^{T} & G
\end{array}\right] \geq 0} \\
& {\left[\begin{array}{cc}
1 & x_{0}^{T} \\
x_{0} & G
\end{array}\right] \geq 0}
\end{aligned}
$$

where:

$$
X=A G+B \operatorname{diag}\left\{\bar{u}_{i}\right\}\left(D_{i} W+D_{i}^{-} V\right),
$$

and $v_{i}$ is the $i$ th row of matrix $V$. Then $u(k)=$ $W G^{-1} x(k)$ is the guaranteed cost controller of system (14) satisfying cost function $J \leq \rho$ and $\varepsilon(P, \rho)$ is a positive invariant set. Furthermore, the HPLF is given by Eq. (20).

Proof: Consider the discrete time system whose guaranteed cost controller is to be developed. Multiplying Eq. (15) by $\rho^{1 / 2} P^{-1}$ on the right and left, respectively, gives:

$$
\begin{aligned}
& \rho P^{-1}\left[A+B \operatorname{diag}\left\{\tilde{u}_{i}\right\}\left(D_{i} K+\bar{D}_{i} H\right)\right]^{T} \\
& P\left[A+B \operatorname{diag}\left\{\tilde{u}_{i}\right\}\left(D_{i} K+\bar{D}_{i} H\right)\right] P^{-1}-\rho P^{-1} \\
& \quad+\rho P^{-1} Q P^{-1}+\rho P^{-1} K^{T} R K P^{-1}<0
\end{aligned}
$$

Let $G=\rho P^{-1}, W=\rho K P^{-1}, V=\rho H P^{-1}$; using the Schur compliment, the above in equality can be written as:

$$
\left[\begin{array}{cc}
-G+\rho^{-1} G Q G+\rho^{-1} W^{T} R W & X^{T} \\
X & -G
\end{array}\right]<0 .
$$

Applying Schur compliment on the above inequality, it can be written as:

$$
\left[\begin{array}{cccc}
-G & G & W^{T} & X^{T} \\
G & -\rho Q^{-1} & 0 & 0 \\
W & 0 & -\rho R^{-1} & 0 \\
X & 0 & 0 & -G
\end{array}\right]<0
$$

which is equal to the inequality given by Eq. (22).

The saturating non-linearity can be substituted by a convex polytope which is:

$$
\rho h_{i} P^{-1} h_{i}^{T} \leq 1
$$

where $h_{i}$ is the $i$ th row of matrix $H$. Utilizing Schur compliment on Eq. (28), it can be written as:

$$
\left[\begin{array}{cc}
1 & h_{i}\left(\frac{P}{\rho}\right)^{-1} \\
\left(\frac{P}{\rho}\right)^{-1} h_{i}^{T} & \left(\frac{P}{\rho}\right)^{-1}
\end{array}\right] \geq 0 .
$$

Substituting $V=\rho H P^{-1}$ in the above equation, it can be written as:

$$
\left[\begin{array}{cc}
1 & v_{i} \\
v_{i}^{T} & G
\end{array}\right] \geq 0
$$

which is equal to the inequality given by Eq. (23). The invariant set ellipsoid is given by:

$$
x^{T} P x \leq \rho .
$$

Taking Schur compliment of the above inequality and substituting $G=\rho P^{-1}$, it can be written as:

$$
\left[\begin{array}{cc}
1 & x_{0}^{T} \\
x_{0} & G
\end{array}\right] \geq 0
$$

which is equivalent to the inequality given by Eq. (24).

So far the LMIs are derived without integration of the Norm-bounded uncertainties. The discrete time system with Norm-bounded uncertainties is:

$$
\begin{aligned}
x(k+1)= & {\left[A+\Delta A+(B+\Delta B) \operatorname{diag}\left\{\bar{u}_{i}\right\}\right.} \\
& \left.\left(D_{i} K+\bar{D}_{i} H\right)\right] x(k) .
\end{aligned}
$$

Theorem 3: For a symmetric positive definite extended matrix $P_{[m]} \in \mathbb{R}^{n \times n}$ and a scalar $\rho \in \mathbb{R}^{+}$, consider the ellipsoid $\varepsilon(P, \rho):=\left\{x \in \mathbb{R}^{n}: x^{\{m\}^{T}} P x^{T} \leq \rho\right\}$ if there exists a scalar $\varepsilon>0$, then a positive definite extended matrix is given by $G_{[m]} \in \mathbb{R}^{n \times n}$ and $V_{[m]}$, $W_{[m]} \in \mathbb{R}^{m \times n}$ such that:

$$
\begin{aligned}
& {\left[\begin{array}{ccccc}
-G & X^{T} & E^{T} & G & W^{T} \\
X & -G+\varepsilon D D^{T} & 0 & 0 & 0 \\
E & 0 & -\varepsilon I & 0 & 0 \\
G & 0 & 0 & -\rho Q^{-1} & 0 \\
W & 0 & 0 & 0 & -\rho R^{-1}
\end{array}\right]<0} \\
& {\left[\begin{array}{cc}
1 & v_{i} \\
v_{i}^{T} & G
\end{array}\right] \geq 0} \\
& {\left[\begin{array}{cc}
1 & x_{0}^{T} \\
x_{0} & G
\end{array}\right] \geq 0}
\end{aligned}
$$


where:

$$
\begin{aligned}
& X=A G+B \operatorname{diag}\left\{\bar{u}_{i}\right\}\left(D_{i} W+D_{i}^{-} V\right), \\
& E=E_{1} G+E_{2} \operatorname{diag}\left\{\bar{u}_{i}\right\}\left(D_{i} W+D_{i}^{-} V\right),
\end{aligned}
$$

and $v_{i}$ is the $i$ th row of the matrix $V$. Then, $u(t)=$ $W G^{-1} x(t)$ is the guaranteed cost control law of the discrete time System (33) satisfying performance index and $J \leq \rho$. Furthermore, $\varepsilon(P, \rho)$ is a positive invariant set.

Proof of Theorem 3 is carried out in the same manner as that of Theorem 2 with only the corporation of the uncertainty matrix defined by Eq. (12).

Theorems 2 and 3 give the sufficient conditions for the existence of guaranteed cost controller via homogenous polynomial Lyapunov function with the guaranteed cost index $J \leq \rho$. The Convex optimization toolbox helps to choose from all the $\varepsilon(P, \rho)$ that satisfies these conditions such that the guaranteed cost is minimized. Now, this problem can be formulated as:

$\min \rho \quad$ s.t $\quad$ Relations $(22)-(24)$

or Relations $(34)-(36)$.

If the above optimization problem has an optimal solution, then $u=W G^{-1} x(k)$ is the optimal guaranteed cost control law of System (33), which satisfies the performance index $J \leq \rho^{*}$.

It is clear that System (37) is a convex optimization problem with LMI constraints.

\section{Results and conclusion}

Example 1: [17] A Discrete time model is given by:

$$
\begin{aligned}
& x(k+1)=\left(A+B K+D\left(E_{1}+E_{2} K\right)\right) x(k), \\
& x(0)=x_{0} .
\end{aligned}
$$

$D, E_{1}$ and $E_{2}$ are constant matrices with compatible dimensions. The system Matrices are given as:

$$
\begin{array}{ll}
A=\left[\begin{array}{cc}
1 & 0 \\
0 & -1.5
\end{array}\right], & B=\left[\begin{array}{l}
0.3 \\
0.5
\end{array}\right], \\
x_{0}=\left[\begin{array}{l}
1 \\
1
\end{array}\right], & D=\left[\begin{array}{cc}
0.1 & 0 \\
0 & 0.1
\end{array}\right], \\
E_{1}=\left[\begin{array}{ll}
0.2 & 0.3 \\
0.1 & 0.4
\end{array}\right], & E_{2}=\left[\begin{array}{ll}
0.3 & 0 \\
0.1 & 1
\end{array}\right], \\
-3 \leq u_{i} \leq 3, & i=1,2 .
\end{array}
$$

The associated performance index of the system is:

$$
J=\sum_{k=0}^{\infty}\left[x^{T}(k) Q x(k)+u^{T}(k) R u(k)\right] .
$$

Using the approach suggested in [9], we obtain the optimal guaranteed cost of the closed-loop system and that is $J^{*}=4.8066$; also, the control law is:

$$
K=\left[\begin{array}{ll}
-0.4260 & 1.7915
\end{array}\right] \text {. }
$$

And the positive definite symmetric matrix $P$ is given by:

$$
P=\left[\begin{array}{cc}
3.0716 & 1.0980 \\
1.0980 & 7.620
\end{array}\right]
$$

While, by applying the proposed method and solving the corresponding optimization problem, we get the optimal guaranteed cost of the system by Eq. (9) and that is $J^{*}=2.3350$; the corresponding control law is:

$$
K=\left[\begin{array}{ccc}
2.4937 & -0.0126 & 0.0279 \\
-0.0021 & 0.0011 & -2.6764 \\
0 & 0 & 0
\end{array}\right]
$$

and the associated homogeneous polynomial Lyapunov is:

$$
\begin{aligned}
v_{2 m}= & 1.2480 x_{1}^{4}+0.0630 x_{1}^{3} x_{2}-0.1085 x_{1}^{2} x_{2}^{2} \\
& +0.0630 x_{1}^{3} x_{2}+0.1280 x_{1}^{2} x_{2}^{2}-0.0626 x_{1} x_{2}^{3} \\
& -0.1085 x_{1}^{2} x_{2}^{2}-0.0626 x_{1} x_{2}^{2}+1.1752 x_{2}^{4} .
\end{aligned}
$$

The positive definite symmetric matrix $P$ is given by:

$$
\begin{aligned}
v_{2 m}= & {\left[\begin{array}{c}
x_{1}^{2} \\
x_{1} x_{2} \\
x_{2}^{2}
\end{array}\right]^{T}\left[\begin{array}{ccc}
1.2480 & 0.0630 & -0.1085 \\
0.0630 & 0.1280 & -0.0626 \\
-0.1085 & -0.0626 & 1.1752
\end{array}\right] } \\
& {\left[\begin{array}{c}
x_{1}^{2} \\
x_{1} x_{2} \\
x_{2}^{2}
\end{array}\right] . }
\end{aligned}
$$

It can be observed that our method gives a lower bound of the guaranteed cost. Furthermore, the invariant set also shows an increase in the radius of convergence of the system.

Example 2: Consider a discrete time system:

$$
\begin{aligned}
& x(k+1)=A x(k)+B u(k), \\
& x(0)=x_{0},
\end{aligned}
$$

with the system matrix:

$$
A=\left[\begin{array}{cc}
-0.5572 & -0.7814 \\
0.7814 & 0
\end{array}\right] \quad B=\left[\begin{array}{cc}
1 & -1 \\
0 & 2
\end{array}\right],
$$




$$
\begin{aligned}
& x_{0}=\left[\begin{array}{l}
1 \\
1
\end{array}\right], \quad Q=\left[\begin{array}{cc}
0.5 & 0 \\
0 & 0.5
\end{array}\right], \quad R=\left[\begin{array}{ll}
1 & 0 \\
0 & 1
\end{array}\right], \\
& -4 \leq u_{i} \leq 4, \quad i=1,2 .
\end{aligned}
$$

The associated performance index of the system is:

$$
J=\sum_{k=0}^{\infty}\left[x^{T}(k) Q x(k)+u^{T}(k) R u(k)\right],
$$

where $Q=\operatorname{diag}\{0.5,0.5\}$, and $R=\operatorname{diag}\{1,1\}$.

Using the traditional quadratic approach [17], the optimal guaranteed cost of the closed-loop system is $J^{*}=1.4164$, while by applying the proposed method (Theorem 2) and solving the corresponding optimization problem, the calculated optimal guaranteed cost of the system by Eq. (9) is $J^{*}=0.4345$. It can be seen from Figure 1 that our approach proves to be more stable for the given set of initial conditions.

It is obvious that our method gives a lower bound of the guaranteed cost. Furthermore, Figure 1 shows a significant increase in the radius of convergence of the system. The associated homogeneous Lyapunov polynomial is:

$$
\begin{aligned}
v_{2 m}= & 0.1772 x_{1}^{4}-0.0393 x_{1}^{3} x_{2}-0.0381 x_{1}^{2} x_{2}^{2} \\
& -0.0393 x_{1}^{3} x_{2}+0.1778 x_{1}^{2} x_{2}^{2}-0.0385 x_{1} x_{2}^{3} \\
& -0.0381 x_{1}^{2} x_{2}^{2}-0.0385 x_{1} x_{2}^{2}+0.1766 x_{2}^{4}
\end{aligned}
$$

and the associated positive definite symmetric matrix $P$ is given by:

$$
P=\left[\begin{array}{ccc}
0.1772 & -0.0393 & -0.0381 \\
-0.0393 & 0.1778 & -0.0385 \\
-0.0381 & -0.0385 & 0.1766
\end{array}\right] \text {. }
$$

Consider a case in which a problem has uncertainty and saturation. A discrete time system with uncertainty

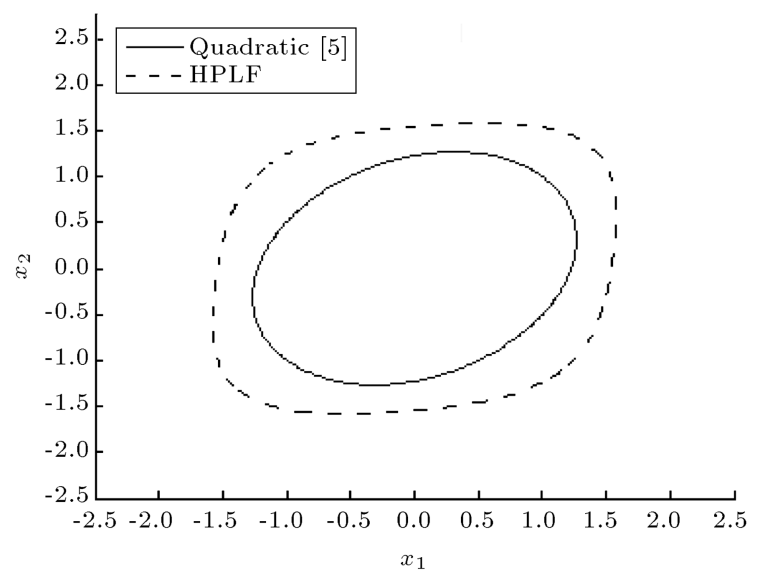

Figure 1. Invariant sets of quadratic and HPLF approaches. and saturation is given by:

$$
\begin{aligned}
& x(k+1)=\left(A+B K+D\left(E_{1}+E_{2} K\right)\right) x(k), \\
& x(0)=x_{0},
\end{aligned}
$$

where $D, E_{1}$ and $E_{2}$ are constant matrices with compatible dimensions. With the system matrix:

$$
\begin{aligned}
& A=\left[\begin{array}{cc}
-0.5572 & -0.7814 \\
0.7814 & 0
\end{array}\right], \quad B=\left[\begin{array}{cc}
1 & -1 \\
0 & 2
\end{array}\right], \\
& x_{0}=\left[\begin{array}{l}
1 \\
1
\end{array}\right], \quad D=\left[\begin{array}{cc}
0.7 & 0 \\
0 & 0.4
\end{array}\right], \\
& E_{1}=\left[\begin{array}{ll}
0.8 & 0.1 \\
0.4 & 0.7
\end{array}\right], \quad E_{2}=\left[\begin{array}{cc}
0.1 & 0.3 \\
0.4 & 1
\end{array}\right], \\
& -3 \leq u_{i} \leq 3, \quad i=1,2,
\end{aligned}
$$

the associated performance index of the system is:

$$
J=\sum_{k=0}^{\infty}\left[x^{T}(k) Q x(k)+u^{T}(k) R u(k)\right],
$$

where $Q=\operatorname{diag}\{0.5,0.5\}$, and $R=\operatorname{diag}\{1,1\}$.

Using the same quadratic approach [9,24-26], the optimal guaranteed cost of the uncertain closed-loop system is $J^{*}=0.8033$. By applying Theorem 3 and solving the corresponding optimization problem, the optimal guaranteed cost of the uncertain closed loop system is $J^{*}=0.4688$. Figure 2 shows the invariant set of both approaches.

It is clear from the results that the proposed approaches prove to be less conservative even in the presence of actuator saturation and uncertainties. However, the presence of uncertainties and actuator saturation does not show a remarkable increase in the radius of convergence as shown in Figure 3.

The significant change in the control input, which is computed by the proposed method and is known as an optimized guaranteed cost control law, is illustrated by Figure 4 .

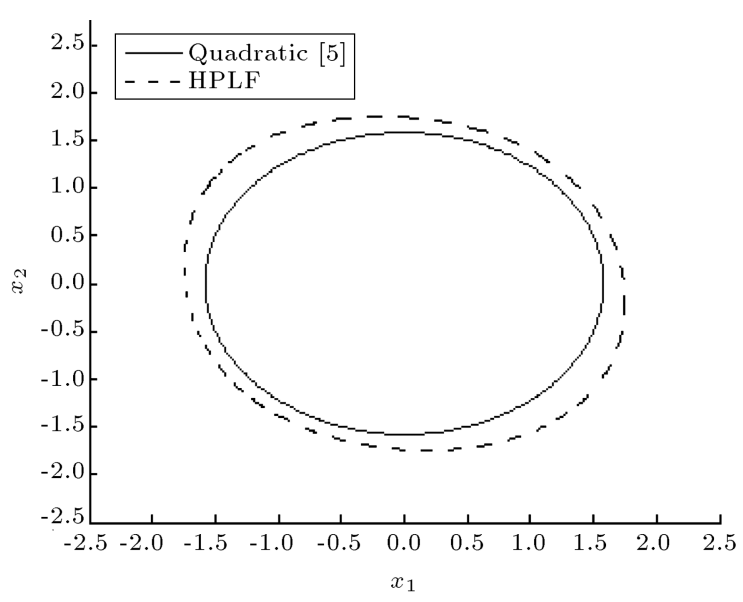

Figure 2. Invariant set of quadratic and HPLF. 


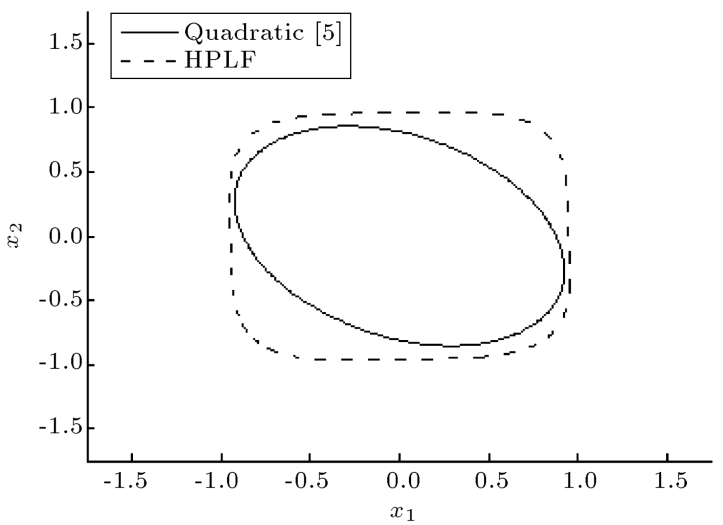

Figure 3. Invariant set of state(s).

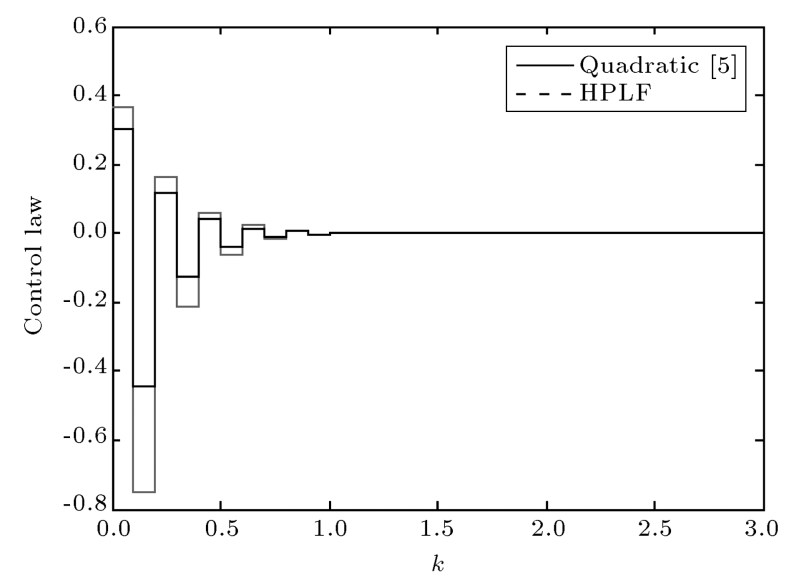

Figure 4. Control law of both approaches.

\section{Nomenclature}

$I_{n} \quad n \times n$ identity matrix

$A^{T} \quad$ Transpose of matrix $A$

$A>0 \quad$ Symmetric positive definite matrix $A$

$\oplus \quad$ Kronecker product

$\|x\| \quad$ Euclidean norm of a vector

(j) Time derivative of a matrix or vector

()$^{*} \quad$ Optimal solution

$\mathbb{R}^{n} \quad \mathbb{R}^{n} n$-dimensional real space

$\operatorname{co}\{$.$\} \quad Convex hull;$

$P \quad$ Matrix $P$ used in Lyapunov function

$\sigma \quad$ Saturation

$V \quad$ Lyapunov function

$u \quad$ Control input

()* Hermitian matrix

\section{References}

1. Chesi, G., Garulli, A. and Vicino, A. "Homogeneous Lyapunov function for systems with structured uncertainties", [Online], Available at www.eee.hku.hk/ chesi/paper
2. Hu, T., Lin. Z. and Chen, B.M. "Analysis and design for discrete-time linear systems subject to actuator saturations", System and Control Letter, 45, pp. 97112 (2002).

3. Boyd, S., Ghaoui, L.E., Feron, E. and Balakrishnan, V., LMI in System and Control Theory, PA: SIAM (1994)

4. Olas, A. "Construction of optimal Lyapunov function for systems with structured uncertainties", IEEE Transaction on Automatic Control, 39, pp. 167-171 (1994)

5. Xie, L. and Soh, Y. "Control of uncertain discrete time systems with guaranteed cost", Proceeding of the 32nd Conference on Decisions and Control, Texas (1993)

6. Sharif Ahmadian, A., Hosseingholi, M. and Ejlali, A. "Discrete feedback-based dynamic voltage scaling for safety critical real-time systems", Scientia Iranica, 20(3), pp. 647-656 (2013)

7. Bekey, G.A. "Analysis and synthesis of discrete-time systems", Scientia Iranica, 18(3), pp. 639-654 (2011)

8. Amato, F., Ambrosino, R., Ariola, M. and Morela, A. "Domain of Attraction and guaranteed cost controller for nonlinear quadratic linear systems", Part: 2, Controller Design, IET Control Theory Appl. (2013).

9. Jarvis, Z. and Packard, A.K. "An LMI method to demonstrate simultaneous stability using non quadratic polynomial Lyapunov functions", Proceeding of the 41 st IEEE Conference on Decision and Control (2002).

10. Amato, F., Ambrosino, R., Ariola, M. and Morela, A. "Domain of attraction and guaranteed cost controller for nonlinear quadratic linear systems", Part: 1, Analysis, IET Control Theory Appl. (2012).

11. Amato, F., Ambrosino, R. and Ariola, M. "Robust stability via polyhedral Lyapunov function", American Control Conference (2009).

12. Johanson, M. and Rantzer, A. "Computation of piecewise quadratic Lyapunov functions for hybrid system", IEEE Transaction on Automatic Control, 43, pp. 555559 (1989).

13. Cao, Y.Y. and Lin, Z. "Stability analysis of discrete time system with actuator saturation by saturation dependent Lyapunov function", Proceeding of the 41 st IEEE Conference on Decision and Control (2002).

14. Yantao, W. and Xian, Z. "HPLF-based robust stability criteria for uncertain continous-time linear system", Proceeding of the 29th Chinese Control Conference (2010).

15. Amato, F., Colacino, D., Cosentino, C. and Morela, A. "Invariant sets and guaranteed cost control for nonlinear quadratic systems", Proceedings of the IEEE MED 2013, Creta Greece (2010).

16. Van Antwerp, J.G. and Braatz, R.D. "A tutorial on linear and bilinear matrix inequalities", Journal of Process Control, pp. 363-385 (2010) 
17. Zuo, Z., Jia, Z., Wang, Y., Zhao, H. and Zhang, G. "Guaranteed cost control for discrete-time uncertain systems with saturating actuators", American Control Conference, Washington, USA (2008)

18. Hindi, H. and Boyd, S. "Analysis of linear systems with saturation using convex optimization", Proceedings of the 37th IEEE Conference on Decision and Control, pp. 903-908 (1998).

19. Yu, L., Han, Q.L. and Sun, M.X. "Optimal guaranteed cost control of linear uncertain system with input constraints", International Journal of Control, Automation, and System, 3(3), pp. 397-402 (2003).

20. Blanchini, F. "Nonquadratic Lyapunov functions for robust control", Automatica, 31, pp. 451-461 (1995).

21. Shekofteh, Y. and Almasganj, F. "Remote diagnosis of unilateral vocal fold paralysis using matching pursuit based features extracted from telephony speech signal", Scientia Iranica, 20(6), pp. 2051-2060 (2013).

22. Hu, T., Andrew, R. and Zaccarian, L. "Stability and performance for saturated systems via quadratic and nonquadratic Lyapunov functions", IEEE Transactions on Automatic Control, 51(11), pp. 1770-1786 (2006).

23. Amato, F., Colacino, D., Cosentino, C. and Morela, A. "Guaranteed cost control for uncertain nonlinear quadractic systems", Proceedings of the ECC, Strasburgo (2014).

24. Wang, C., Chen, Z.W. and Chen, G.Y. "Delaydependent passive analysis and control for interval stochastic time-delay systems", Scientia Iranica, 21(3), pp. 628-646 (2014)

25. Bolandi, H., Haghparast, M. and Abedi, M. "A reliable fault tolerant attitude control system based on an adaptive fault detection and diagnosis algorithm together with a backstepping fault recovery controller", Scientia Iranica, 20(6), pp. 1999-2014 (2013)

26. Ogata, K., Discrete-Time Control Systems, 2nd Ed., Prentice-Hall India (1995).

\section{Biographies}

Syed Muhammad Nashit Arshad completed his high school in a Government college. After high school, he went to Air University to get the bachelor degree in Mechatronics as he leant towards robotics from his childhood. After successful completion of his bachelor degree, he preferred to gain more knowledge about the control system used in Mechatronics. He started his master in Mechatronics with specialization in control systems. He served various industries as control engineer, design engineer, and production engineer. Nowadays he serves as a lecturer in Electronics Department, Iqra University, Islamabad, Pakistan, and has also started his PhD in Rrobotics with specialization in artificial intelligence at NUST
Islamabad. He loves to make robots and remote control aeroplanes. His hobbies include aero modeling and traveling.

Kamal Mazhar completed his high school in a Cadet College, Kalar Kahar, Pakistan. After high school, he went to Air University to get the bachelor degree in Mechatronics as he was interested in robotics and AI. After successful completion of his bachelor degree, he preferred to gain more knowledge about automobiles in continuation of his final year project. He started his master in Mechanical with specialization in control systems. He is a control engineer with 5 years of industrial experience. He is a member of controls and signal processing group (caspr) with strong research interest in automotive parameter estimation and controller/observer implementation. His hobbies include reading, traveling, and $\mathrm{RC}$ planes.

Umer Khan graduated with a degree in Computer Science from International Islamic University in 2004. He then pursued PhD in Electrical Engineering from 2011 at Pakistan Institute of Engineering and Applied Sciences. From 2011 to 2014, he also served as an Assistant Professor at the Department of Mechatronics, Air University. He has also one year of experience as a Postdoctoral Research Fellow at the Department of Computing, The Hong Kong Polytechnic University, in 2014-2015. His research interest includes robust control, linear matrix inequalities, vision-based control, and optimal control.

Syed Muhammad Usman Hashmi has obtained his Electrical Engineering degree from Federal Urdu University of Arts, Science and Technology (FUUAST), Islamabad, Pakistan, in 2008. He obtained his MS (Telecommunication and Networks) degree with distinction (Gold-Medalist) from Iqra University, Islamabad, Pakistan, in 2010. During the course of his studies, he was actively involved in various research activities. He worked on error correction and coding schemes (e.g., Reed-Solomon codes and LDPC codes) and many implementations in the context of different neural networks. After his MS, he remained attached with a mobile application development organization and worked on server side using VB.NET. Later, he joined Iqra University as lecturer in 2011. He worked on DSP kits and simulated physical layer and application layer time synchronization of Wireless Sensor Network. A novel cross-layer design for time synchronization that proves to be energy efficient has been proposed. Parallel to this contribution, he is working on cluster head selection of Wireless Sensor Network using artificial intelligence techniques, control systems, and security issues. 